\title{
Effects of vertical vibration on hopper flows of granular material
}

\author{
C. R. Wassgren ${ }^{\text {a) }}$ \\ School of Mechanical Engineering, Purdue University, West Lafayette, Indiana 47907-1288 \\ M. L. Hunt, P. J. Freese, J. Palamara, and C. E. Brennen \\ Division of Engineering and Applied Science, California Institute of Technology, Pasadena, California 91125
}

(Received 30 August 2001; accepted 9 July 2002; published 3 September 2002)

\begin{abstract}
The discharge of granular material from a hopper subject to vertical sinusoidal oscillations was investigated using experiments and discrete element computer simulations. With the hopper exit closed, side-wall convection cells are observed, oriented such that particles move up along the inclined walls of the hopper and down at the center line. The convection cells are a result of the granular bed dilation during free fall and the subsequent interaction with the hopper walls. The mass discharge rate for a vibrating hopper scaled by the discharge rate without vibration reaches a maximum value at a dimensionless velocity amplitude just greater than 1 . Further increases in the velocity decrease the discharge rate. This decrease occurs due to a decrease in the bulk density of the discharging material when vibration is applied. () 2002 American Institute of Physics. [DOI: $10.1063 / 1.1503354]$
\end{abstract}

\section{INTRODUCTION}

Hoppers and bins are routinely used to transport, process or store bulk materials such as coal, ore, grain, and plastics. Although hoppers are common, the internal flow of the material is not well understood, relying heavily on empirical information to maintain operation. For example, when a batch of material is introduced into a hopper for the first time, the material at the exit may arch and prevent flow. To remedy the situation, vibration may be used, sometimes in the crude form of a hammer, to perturb the material and initiate the flow. Alternatively, the hopper may be equipped with a bin activator to continuously shake part of the hopper wall. These bin activators must be carefully designed to enhance the flow and not result in further settling or clogging of the material.

The focus of this study is to examine through experiments and discrete element simulations the effect of vertical vibration on flow from a planar hopper. This study is a companion paper to the recent work by Hunt et al. ${ }^{1}$ on the effects of horizontal vibration on hopper discharge. The earlier work demonstrated that horizontal vibration increased the mass discharge rate as compared with the discharge rate from a hopper without vibration and that the increase depended on the vibration velocity amplitude. In addition, the discharging granular material flows from alternating sides of the hopper producing an inverted funnel pattern.

Over the last decade, the effect of vertical vibration on a bed of material has been studied extensively. For a box that is vibrated sinusoidally, $z=a \sin (\omega t)$ where $a$ is the amplitude of vibration and $\omega$ is the radian frequency, the bed exhibits several different flow patterns depending on the dimensionless acceleration amplitude, $\Gamma=a \omega^{2} / g$ where $g$ is the gravitational constant, and the frequency, $f=\omega /(2 \pi)$. For $\Gamma>1$, side-wall convection cells appear where particles

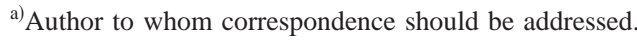

move down along vertical walls of the container and up within the remainder of the bed. Standing waves, forming at one-half the forcing frequency, appear on the free surface of the bed for $2.2<\Gamma<3.5$ and waves forming at one-quarter the forcing frequency occur for $\Gamma>5.5$. Neighboring regions of the particle bed can oscillate out-of-phase, termed "kink waves," for $\Gamma>3.5$ with counter-rotating "kink convection cells" bracketing each wave node. The paper by Wassgren et $a .^{2}$ describes these phenomena in greater detail.

The effects of vertical vibration on flow from wedgeshaped hoppers and flat bottom bins were first examined by Takahashi et al. ${ }^{3}$ and Suzuki et l. $^{4}{ }^{4}$ These studies reported the appearance of convection cells near the inclined wall boundaries of the hopper. In addition, the discharge rate was shown to increase with vibrational frequency at a fixed acceleration level, but that at the highest accelerations the discharge rate decreased significantly. Vibration also induced flow in bins that could not discharge under gravity alone. Lindemann and Dimon ${ }^{5}$ recently investigated flow from vertically oscillated funnels with small exit widths and wall angles (as measured from the hopper centerline). They found that the dimensionless acceleration amplitude, $\Gamma$, and wall angle significantly affect how particles "jam" or mechanically arch at the exit. Lindemann and Dimon ${ }^{5}$ also report that the flow rate from vibrated hoppers decreases with increasing $\Gamma$. Knight et al., ${ }^{6}$ although not investigating hopper flows specifically, also reported the appearance of convection cells in experiments using vertically oscillating conical containers. More recently, Evesque and Meftah $^{7}$ examined the time required to discharge a known quantity of sand from a sealed vertically vibrating hourglass and observed that the discharge rate decreases with increasing acceleration amplitude.

Without vibration, the mass discharge rate from a hopper, $W$, is proportional to the bulk density of the bed near the hopper exit, $\rho_{b}$, the square root of the acceleration acting on the bed, $g$ (the acceleration due to gravity), and the hydraulic diameter of the hopper exit, $D_{h}$, raised to the $5 / 2$ power: 


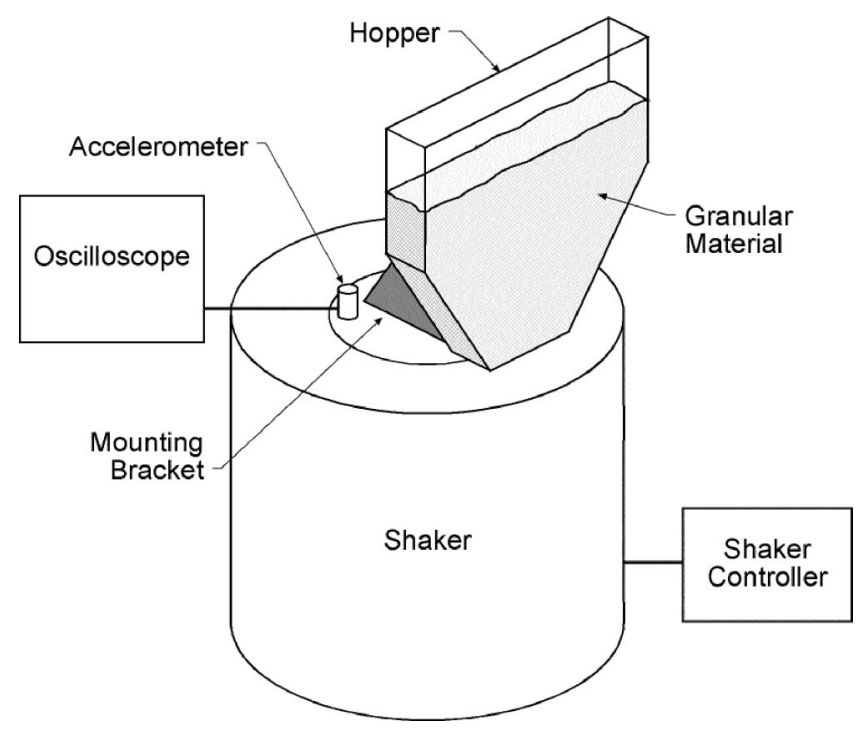

FIG. 1. A schematic of the experimental apparatus.

$$
W \propto \rho_{b} g^{1 / 2} D_{h}^{5 / 2} .
$$

To predict trends in the discharge rate with vibration, Suzuki et $a{ }^{4}{ }^{4}$ proposed a simple model that includes the variation of the "effective gravity" acting on the granular material over an oscillation cycle. Since the hopper is oscillating, the effective gravity the bed experiences relative to the hopper walls, $g_{\text {eff }}$, will vary throughout an oscillation cycle as $g_{\text {eff }}$ $=g[1-\Gamma \sin (\omega t)]$ for $\Gamma \leqslant 1$. If the acceleration amplitude of the oscillations is greater than one $(\Gamma>1)$ the bed leaves the hopper walls during a portion of the oscillation cycle and contacts the walls at some later time. The equations originally derived by Suzuki et al. ${ }^{4}$ also included an empirically derived expression for the bulk density of the bed as a function of $\Gamma$.

This paper examines the particle trajectories and discharge rates from a vertically oscillating, wedge-shaped hopper using both experiments and two-dimensional discrete element computer simulations. First the experiments are described and qualitative and quantitative results are presented. Next, results from discrete element computer simulations are presented. Last, these results are compared with a model originally proposed by Suzuki et al. ${ }^{4}$ to predict the mass discharge rate from a vertically oscillating hopper.

\section{EXPERIMENTS}

The experimental apparatus consisted of a planar, wedge-shaped hopper filled with $1.3 \mathrm{~mm}$ diameter soda-lime glass spheres. The hopper was mounted on an electromagnetic shaker that subjected the entire hopper apparatus to vertical, sinusoidal oscillations. A schematic of the apparatus is shown in Fig. 1.

Two different hoppers (an MB Dynamics and a Ling A-175) were used in the experiments. The front and rear walls of the first hopper, referred to hereafter as hopper I, were lined with smooth window glass; the $45^{\circ}$ side walls were made of Plexiglass with a smooth milled finish. The distance, $C$, between the front and back walls was $12.7 \mathrm{~mm}$ (approximately 10 particle diameters) and the exit width, $B$, was $4.0 \mathrm{~mm}$ giving an exit hydraulic diameter, $D_{h}$ $=(B C)^{1 / 2}$, of approximately $7.1 \mathrm{~mm}$. The second hopper, or hopper II, had a depth of $12.4 \mathrm{~mm}$ and an exit width of 9.6 $\mathrm{mm}\left(D_{h}=10.9 \mathrm{~mm}\right)$; the front and back walls were Plexiglass. The experiments with the glass walls of hopper I did not appear to be affected by static electricity. With the Plexiglass walls of hopper II, some particles did adhere to the walls after the bed was emptied; however, the amount of material remaining on the walls was much less than the total amount discharged.

The vibration frequency and acceleration were monitored using accelerometers mounted to the base plate of the shakers. For hopper I, the frequencies, $f$, examined in the experiments ranged from 20 to $60 \mathrm{~Hz}$ and the dimensionless acceleration amplitude of the oscillations, $\Gamma=a \omega^{2} / g$, ranged from 0 to 4.0. For hopper II, the range was larger, with frequencies from 5 to $80 \mathrm{~Hz}$, and dimensionless accelerations up to $\Gamma=8.0$.

In all of the experiments, the hoppers were filled with 200-250 g of particles so that the initial height of the free surface was at least $10 D_{h}$. The average discharge rate was determined by recording the time required for the hopper to completely discharge a known mass of material. Without vibration, the instantaneous discharge rate was approximately constant except for a short transient when the hopper exit is first opened, and a final transient when the free surface height of the material above the exit is approximately equal to the exit diameter. ${ }^{8}$

\section{A. The flow field}

To follow the trajectories of particles within the hopper, some of the clear glass spheres were dyed black. The nonvibrating hopper displayed a typical funnel flow pattern as it discharged where a region of stagnant material appeared adjacent to each inclined hopper wall. The free surface of the material formed a V-shaped valley with particles continuously avalanching down the sloped surfaces toward the center line of the hopper. As the height of the free surface from the exit plane decreased, the stagnant regions became smaller until all of the material in the hopper flowed.

With vibration, the flow pattern depended on the magnitude of the vibrational acceleration. With the hopper exit closed for $\Gamma>1$, two convection cells appeared with particles moving up in a narrow boundary layer along the inclined walls of the hopper and down at the center line of the hopper. These convection cells were similar, but in the opposite direction, to the convection cells observed in granular beds subject to vertical oscillations in containers with vertical walls. ${ }^{6,9}$ Surface waves forming at one-half the oscillation frequency (referred to as $f / 2$ waves) also appeared on the free surface of the bed when the dimensionless acceleration amplitude, $\Gamma$, was between 2.5 and 4 ; these waves are similar to those observed in experiments of vibrating granular beds. ${ }^{2,10}$ Figure 2 shows photographs of the surface waves with the hopper illuminated from the back.

When the exit of the hopper was opened the circulation patterns continued as the hopper discharged. Figure 3 shows a sequence of images in which the hopper is filled with al- 


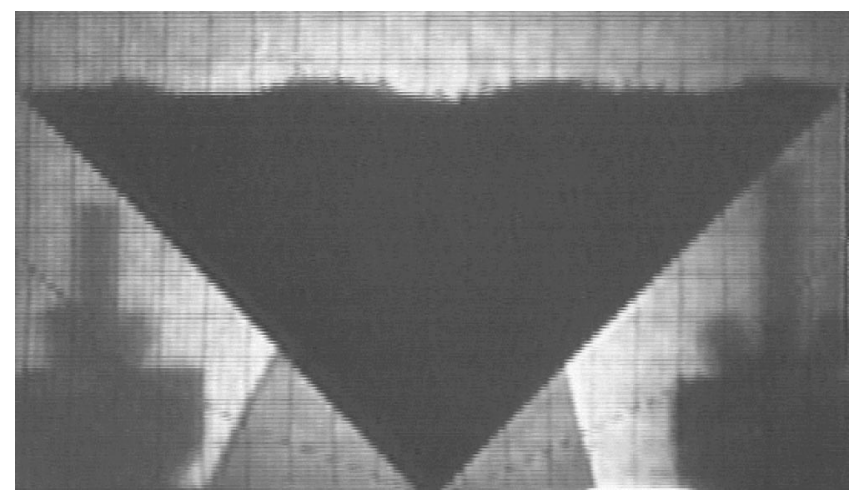

FIG. 2. Photograph of a back-lit, vibrating hopper showing the formation of $f / 2$ surface waves for $\Gamma=3.4$ and $f=30 \mathrm{~Hz}$.

ternating layers of clear and dyed beads. The images are taken at $\Gamma=6$ and $f=10 \mathrm{~Hz}$. At $t=10 \mathrm{~s}$ the hopper is opened and the material begins to discharge. The material continues to circulate as the hopper discharges until at $t$ $=21.8 \mathrm{~s}$ the hopper is completely emptied. The $f / 2$ surface waves also continue to form as the material discharges from the hopper until the height of the free surface of the bed from the hopper exit is approximately five exit widths.

Note that the convection strength and direction depend strongly on the hopper wall angle. ${ }^{11,12}$ For wall angles less than approximately $10^{\circ}$ (measured with respect to the vertical) the convection cells are oriented downward at the walls while for angles greater than $10^{\circ}$ particles move up at the
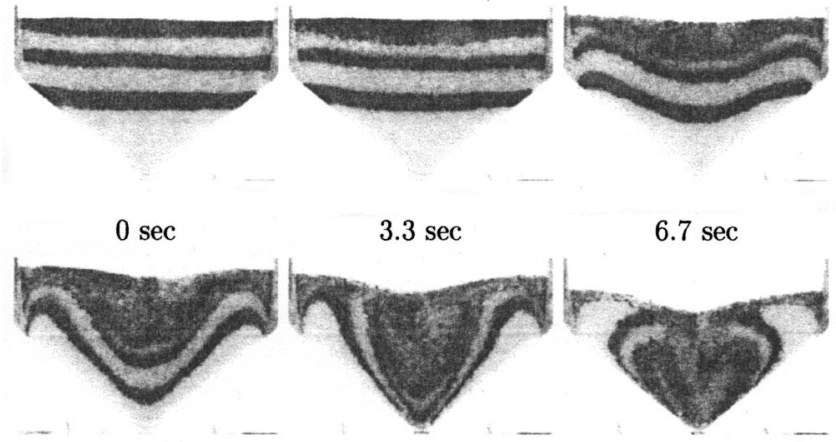

$3.3 \mathrm{sec}$
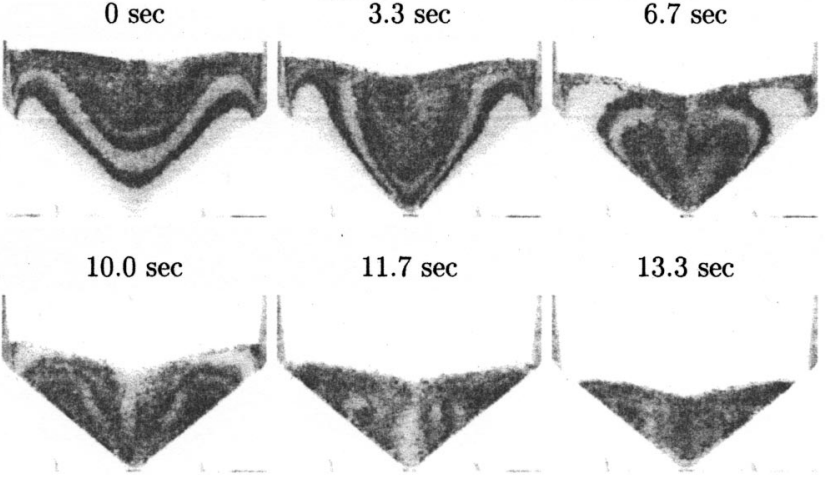

$11.7 \mathrm{sec}$
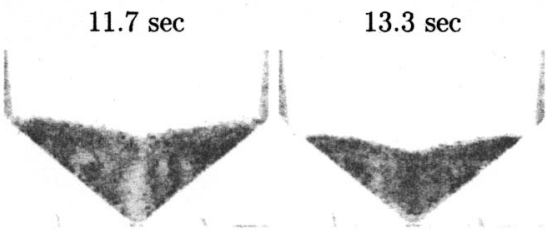

$15.0 \mathrm{sec}$

$16.7 \mathrm{sec}$

$18.3 \mathrm{sec}$

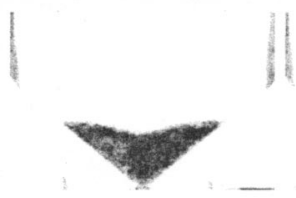

$20.0 \mathrm{sec}$
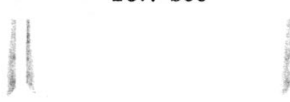

$21.8 \mathrm{sec}$

FIG. 3. A sequence of photographs of a vibrating hopper $(\Gamma=6, f=10 \mathrm{~Hz})$ showing the formation of side-wall convection cells near the inclined walls of the hopper. The hopper is initially closed but is opened at $t=10 \mathrm{~s}$. The hopper discharges completely at $t=21.8 \mathrm{~s}$.

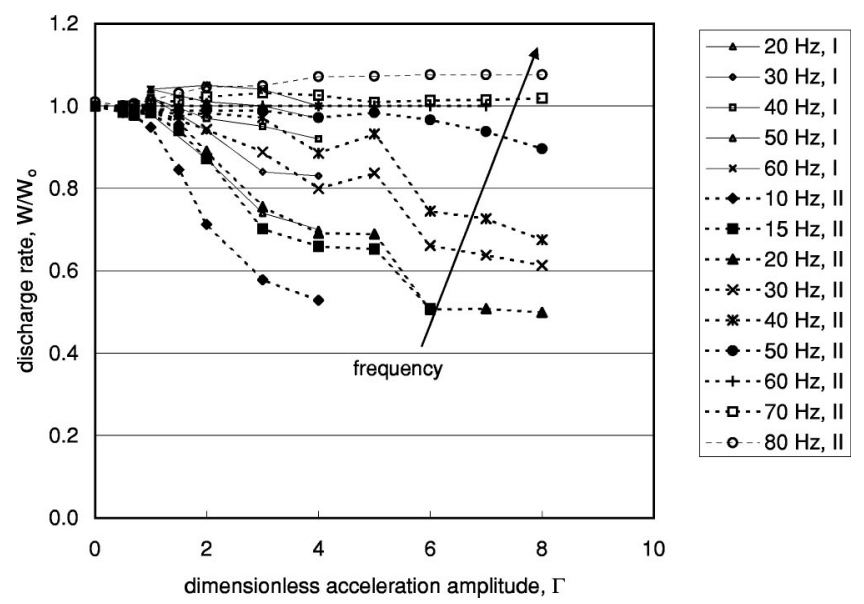

FIG. 4. The ratio of the mass discharge rate from an oscillating hopper, $W$, divided by the mass discharge rate from a nonvibrating hopper, $W_{0}$, plotted as a function of the dimensionless oscillation acceleration amplitude, $\Gamma$ $=a \omega^{2} / g$.

walls. Here only a single wall angle of $45^{\circ}$, for which the convection cells were oriented upward at the walls, was investigated.

\section{B. Mean discharge rates}

The mean discharge rate from the hopper, $W$, was measured over the range of frequencies and accelerations. The discharge rates are normalized by the mean discharge rate for the nonvibrating hopper, $W_{0}$, and are presented in Fig. 4 as a function of dimensionless oscillation acceleration amplitude, $\Gamma=a \omega^{2} / g$, and in Fig. 5 as a function of dimensionless oscillation velocity amplitude, $a \omega /\left(g D_{h}\right)^{1 / 2}$. Each experimental data point in the figures represents the average of five to eight measurements. The difference in discharge rate from experiment to experiment was at most $3 \%$; the uncertainty in the discharge rate was approximately 5\% due to the technique for measuring the discharge time and the loss of material due to static charge.

As shown in Fig. 4, there does not appear to be any significant difference between the data obtained with the two

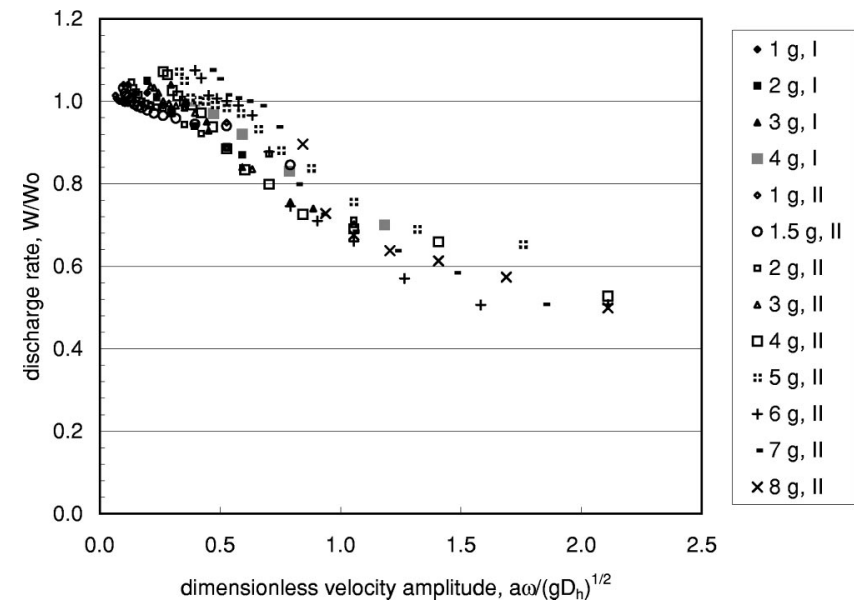

FIG. 5. Mass discharge rate from an oscillating hopper divided by the mass discharge rate from a nonvibrating hopper, $W / W_{0}$, plotted as a function of the dimensionless oscillation velocity amplitude, $a \omega /\left(g D_{h}\right)^{1 / 2}$. 
different hoppers. The discharge rate, $W / W_{0}$, for frequencies below $50 \mathrm{~Hz}$ decreases with increasing acceleration level. At a fixed acceleration, the decrease is most significant at the lowest frequencies. For the highest frequencies $(60$ and 80 $\mathrm{Hz}$ ), the dimensionless discharge rate is approximately equal to or slightly greater than unity.

Figure 5 indicates that the dimensionless discharge rate scales more closely with the velocity amplitude than with the acceleration amplitude. This result was also found in the earlier studies on horizontal vibration. ${ }^{1}$ Again, the discharge rates above unity correspond with the frequencies between 60 and $80 \mathrm{~Hz}$. For frequencies lower than $60 \mathrm{~Hz}$, the data show a monotonic decrease with vibrational velocity with a secondary dependence on acceleration level. At the largest dimensionless velocity amplitude investigated, $a \omega /\left(g D_{h}\right)^{1 / 2}$ $\approx 2.0$, the discharge rate ratio is approximately 0.5 .

\section{Velocities of exiting particles}

The hopper exit velocity was measured for different frequencies and accelerations by recording the flow through the front surface of the hopper at a rate of 2000 frames per second using a Redlake MotionScope camera. Particle tracking ${ }^{13}$ is used to determine the particle velocities near the exit plane. By maximizing the number of pixels per particle, the error in the measurements is approximately $2 \%$.

The velocity of the exiting particles was obtained by averaging over particles within 2 diameters above the hopper exit plane. Hence, the interrogation area moved with the sinusoidally oscillating hopper exit with the neutral position taken at the beginning of the oscillation cycle. The data were also averaged over several cycles of vibration with the spatial and temporal averaged velocity calculated in time intervals of $0.1 / f$. Each data point represents an average over approximately 100-200 particle trajectories. The root-meansquare (rms) velocity fluctuations over an oscillation cycle were also calculated.

Figure 6 presents the normalized velocity measurements as a function of the oscillation cycle at $\Gamma=1.0$ for 10,20 , and $40 \mathrm{~Hz}$. The values are normalized by the cycle-averaged

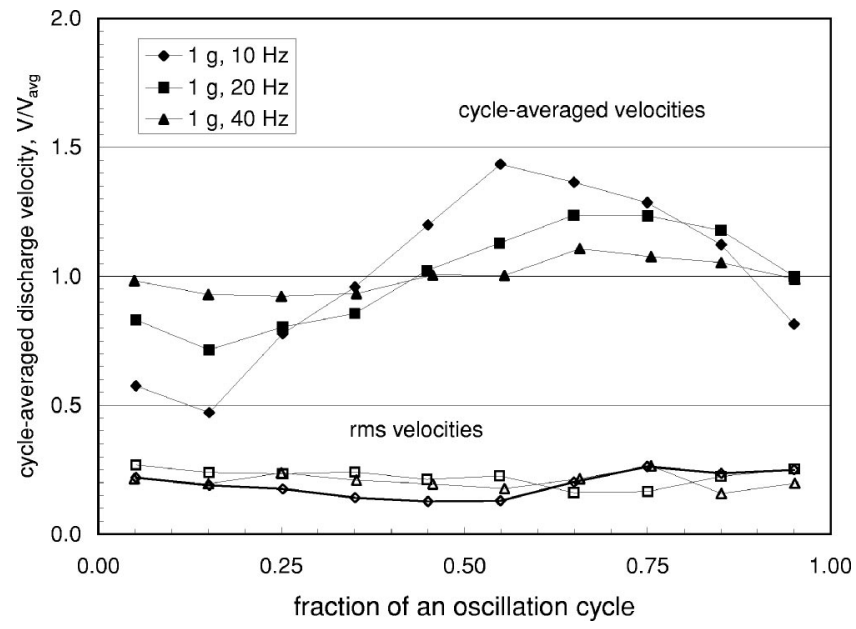

FIG. 6. Discharge velocity of particles relative to the hopper exit normalized by the cycle-averaged velocity and rms velocity as a function of oscillation cycle. Data are shown for $\Gamma=1.0$ and $f=10,20$, and $40 \mathrm{~Hz}$.

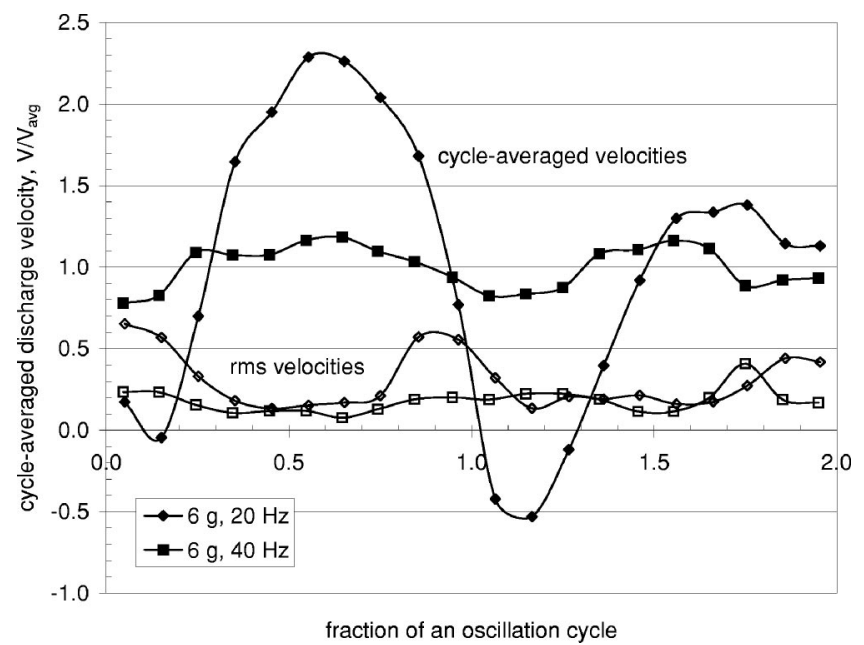

FIG. 7. Discharge velocity of particles relative to the hopper exit normalized by the cycle-averaged velocity and rms velocity as a function of oscillation cycle. Data are shown for $\Gamma=6.0$ and $f=20$ and $40 \mathrm{~Hz}$.

velocity. The results show that normalized velocities vary sinusoidally with a phase lag that depends on the vibrational frequency. At $40 \mathrm{~Hz}$, the phase lag is almost $\pi$, but for 10 $\mathrm{Hz}$, the phase lag is smaller, approximately 0.6 . The amplitude of the normalized velocity also depends on frequency. At $10 \mathrm{~Hz}$, the maximum velocity amplitude is approximately $50 \%$ of the average value; at $40 \mathrm{~Hz}$ the amplitude is approximately $10 \%$ of the average value. Figure 6 also presents the normalized rms velocity fluctuations as a function of the oscillation cycle. Although there is some variation over the cycle, the values remain at approximately $20-25 \%$ of the average velocity. Note that these fluctuations are averaged over the width of the hopper exit. Similar trends are observed for $\Gamma=3.0$ at 20,40 , and $60 \mathrm{~Hz}$.

The velocity data for $\Gamma=6.0$ for 20 and $40 \mathrm{~Hz}$, shown in Fig. 7, are plotted over two cycles of vibration since this acceleration is beyond the period-doubling bifurcation acceleration observed in earlier studies. ${ }^{2}$ The variation in the discharge velocity between the two cycles is clearly observed in the data for $20 \mathrm{~Hz}$. The figure also shows that over part of the cycle, the hopper is moving down faster than the discharging material; hence, the material actually re-enters the hopper.

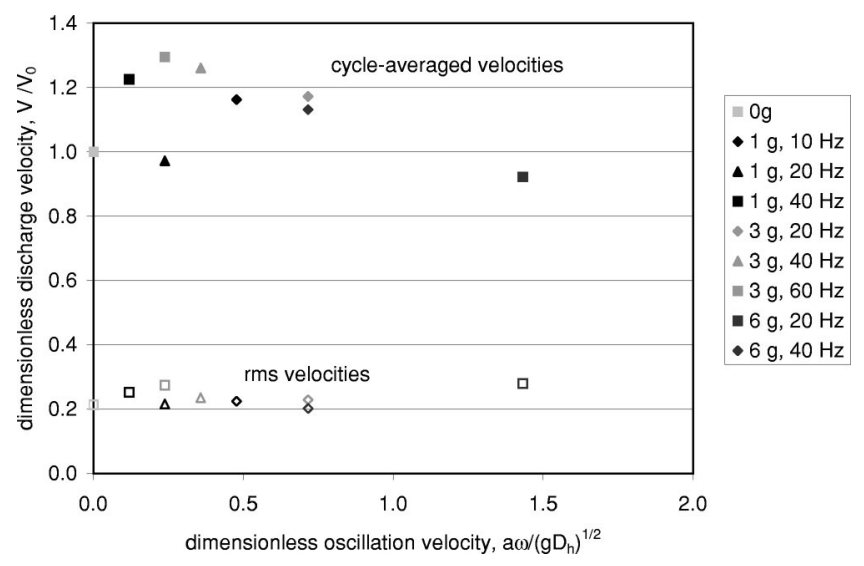

FIG. 8. Cycle-averaged discharge and rms velocities normalized by the nonvibrating hopper discharge velocity as a function of dimensionless velocity amplitude. 
TABLE I. The parameters used in the computer simulation for the vertically oscillating closed hopper.

\begin{tabular}{cc}
\hline \hline Parameter & Value \\
\hline Particle diameter distribution & $0.8-1.2 \mathrm{~mm}$ (uniform distribution) \\
Particle density & $2500 \mathrm{~kg} / \mathrm{m}^{3}$ \\
Exit width/mean particle diameter & 25 \\
Wall angle measured from the centerline & $65^{\circ}$ \\
Number of particles & 513 \\
Coefficient of restitution for particle/particle contacts & 0.9 \\
Friction coefficient for particle/particle contacts & 0.5 \\
Coefficient of restitution for particle/wall contacts & 0.9 \\
Friction coefficient for particle/wall contacts & 1.0 \\
\hline \hline
\end{tabular}

The fluctuation velocities are also significantly larger than those observed at the smaller accelerations. At $40 \mathrm{~Hz}$, the differences over the two cycles of vibration are less evident.

Figure 8 presents the cycle-averaged discharge and rms velocities at $\Gamma=0,1,3$, and 6 normalized by the discharge velocity measured for a nonvibrating hopper. The cycleaveraged velocities are within $25 \%$ of the value for no vibration. In addition, the cycle-averaged rms velocities are of the same magnitude. Hence, vibration of the hopper does not significantly affect the average discharge velocity of the material.

\section{DISCRETE ELEMENT COMPUTER SIMULATIONS}

A two-dimensional soft-particle discrete element simulation was also used to study the vibrated hopper phenomena. The granular material in the simulation consists of spherical particles with a uniform distribution of diameters between a specified minimum and maximum diameter. Each particle has two translational and one rotational degree of freedom. The contact model uses a damped linear spring in the normal direction and a linear spring in series with a frictional sliding element in the tangential direction. ${ }^{14}$ The coefficient of restitution ranges from 0.90 to 0.95 for both the particle-toparticle and the particle-to-wall contacts. The friction coefficient for the particle-to-particle contacts is 0.5 , and for the particle-to-wall contacts is either 0.5 or 1.0. The larger values of the friction coefficient increased the net circulation within the bed. The particles have a density of $2500 \mathrm{~kg} / \mathrm{m}^{3}$ and an average diameter of $3 \mathrm{~mm}$. The details of the simulation parameters can be found in Wassgren. ${ }^{9}$

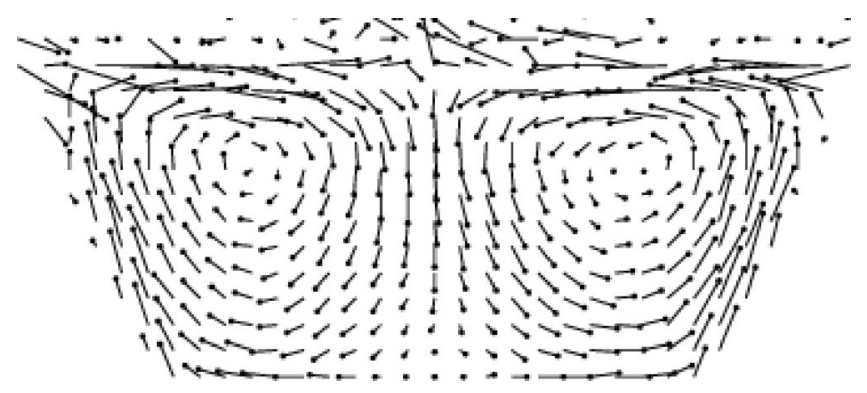

FIG. 9. Side-wall convection cells in a simulated vertically oscillating hopper with a closed exit. The vectors indicating the net displacement of particles per oscillation cycle are shown. The displacements are averaged over 20 oscillation cycles and the circles are the vector arrowheads.

\section{A. Convection in a closed hopper}

The first series of simulations were performed for a container with inclined walls and a closed exit using 513 particles at $f=2.0$ and $5 \mathrm{~Hz}$. The parameters for this simulation are listed in Table I. Two convection cells similar to those observed in the experiments appear as shown in Fig. 9. Particles move up along the inclined walls of the container and down at the center line. When the particle bed leaves the hopper floor, few particle collisions occur with the hopper walls since the walls slope away from the bed and the bed remains closely packed as shown in Fig. 10(a). Thus, the walls apply no force to the particle bed as the bed moves up relative to the hopper. While the bed is in flight it dilates since it is no longer constrained by the container walls. When the dilated bed falls back toward the hopper floor it contacts the inclined walls of the container prior to impacting the base as shown in Fig. 10(b). Due to friction, the walls retard the downward motion of particles near the wall as compared to the remainder of the bed. The resulting net motion of particles near the container walls over an oscillation cycle is upwards along the inclined walls. This same mechanism can also explain the downward motion of particles along vertical walls in vertically vibrated granular beds. ${ }^{9}$

\section{B. Discharge from a stationary hopper}

The flow of granular material from the simulated hopper was also examined. Since the particles are not "recycled" in the simulations, a particle is eliminated from the calculation once it reaches a distance of five hopper widths below the exit plane. The simulation uses a sufficient number of par-

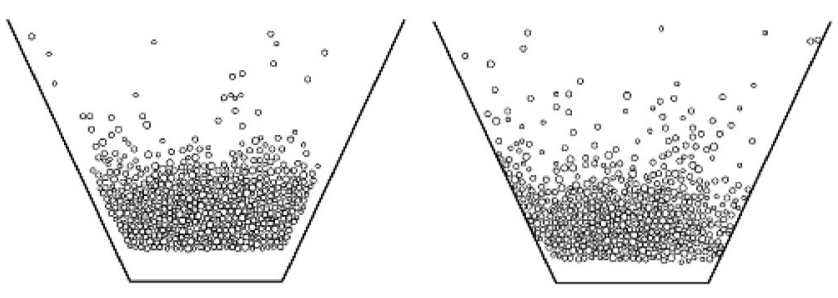

(a) $\phi /(2 \pi)=0.30$

(b) $\phi /(2 \pi)=0.80$

FIG. 10. Snapshots from a simulation of a vertically oscillating hopper $(\Gamma$ $=2.0, f=5 \mathrm{~Hz}$ ) with a closed exit at a phase angle of (a) $\phi /(2 \pi)=0.30$ (bed moving up relative to the hopper walls) and (b) $\phi /(2 \pi)=0.80$ (bed moving down relative to the walls). 
TABLE II. The parameters used in the computer simulations for the vertically oscillating open hopper.

\begin{tabular}{cc}
\hline \hline Parameter & Value \\
\hline Particle diameter distribution & $2.8-3.2 \mathrm{~mm}$ \\
Particle density & $2500 \mathrm{~kg} / \mathrm{m}^{3}$ \\
Exit width/mean particle diameter & 11 \\
Wall angle measured from the centerline & $45^{\circ}$ \\
Number of particles & 10000 \\
Coefficient of restitution for particle/particle contacts & 0.95 \\
Friction coefficient for particle/particle contacts & 0.5 \\
Coefficient of restitution for particle/wall contacts & 0.95 \\
Friction coefficient for particle/wall contacts & 0.5 \\
\hline \hline
\end{tabular}

ticles $(N=10000)$ to ensure that a steady flow is achieved. The remainder of the simulation parameters are given in Table II.

When a particle crosses the horizontal plane of the hopper exit, the time, the horizontal position of the particle, the particle's translational and rotational velocities, and the particle's radius and mass are recorded. The mean discharge rate from the hopper is measured by counting the total number of particles discharged from the hopper between a specified start and end time. The start and end times are chosen such that the initial and final transient periods are avoided. The flow properties are computed across the exit of the channel by dividing the exit into 10 equal length segments. If a particle lies in two adjacent sections, the properties are weighted by the fraction of the particle mass within that section. Note that the exit is 11 particle diameters in width.

Figure 11 presents the mass flow rate, the velocity, and the bulk density as a function of position across the exit of a nonvibrating hopper. The values are normalized by the values averaged over the width. The bulk density of a section is calculated from the mass flow rate divided by the average velocity in that section and the corresponding section width. Note that the maximum solid fraction of a monolayer of particles across the hopper exit is $\pi / 6$. The solid fraction calculated from the simulations is $84 \%$ of the maximum

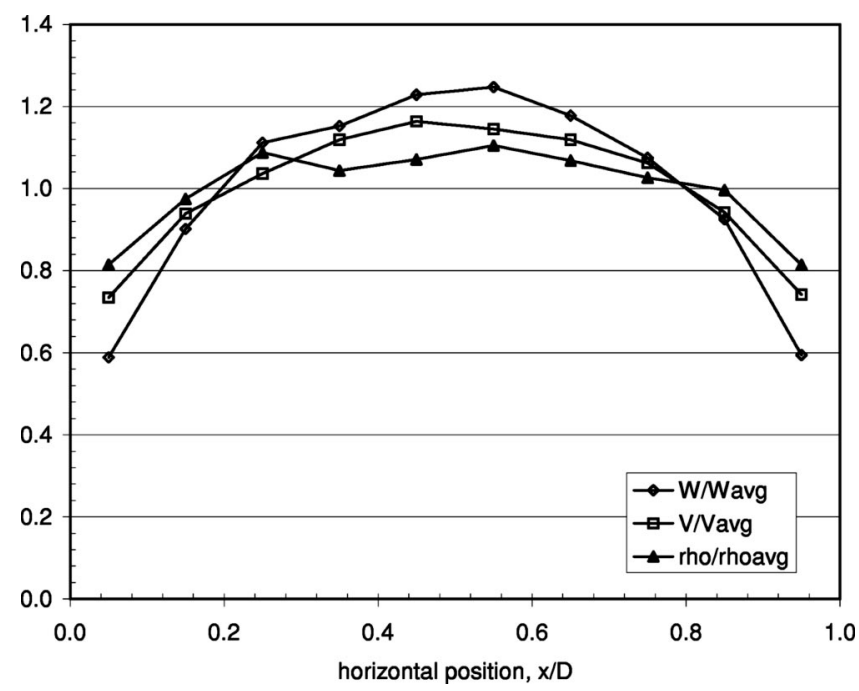

FIG. 11. Spatial distribution of the mass flow rate, $W$, particle velocity, $V$, and bulk density, $\rho_{b}$, across the exit plane of a simulated nonvibrating hopper. The quantities are normalized by their average over the exit plane. value. The average discharge velocity is $1.3\left(g D_{h}\right)^{1 / 2}$, so that the mass flow rate is $W_{0}=1.3 \rho_{b}\left(g D_{h}\right)^{1 / 2} A$ where $D_{h}$ is taken as the width of the hopper exit $(11 d)$ and $A=11 d^{2}$.

\section{Discharge rates with vibration}

The ratio of the mass discharge rate to the mass discharge rate for the nonvibrating hopper, $W / W_{0}$, is plotted in Fig. 12 as a function of amplitude oscillation velocity $a \omega /\left(g D_{h}\right)^{1 / 2}$ for $0<\Gamma<5$ and for frequencies from 5 to 60 Hz. The simulation data show the same trends as the data gathered from the experiments. If the data are plotted as a function of the acceleration amplitude, the simulation results appear to follow the experimental trends indicating that the dimensionless discharge rate was closer to unity for higher frequencies $(50$ and $60 \mathrm{~Hz})$ at a fixed value of the acceleration amplitude.

Besides the average discharge rate, the simulations were used to determine other properties of the flow. In Fig. 13 the discharge rate as a function of phase angle $(\Delta \phi=2 \pi / 10)$ is presented at a frequency of $20 \mathrm{~Hz}$. The data are obtained by mass-averaging over 70 oscillation cycles. The values are normalized by the discharge rate without vibration. As the acceleration amplitude increases, the ratio of the discharge rates show deviations from unity over the cycle of vibration. As the hopper begins its upward motion $[0<\phi /(2 \pi)$ $<0.25]$, the ratio is greater than unity and the material is discharging faster than it would without vibration. As the

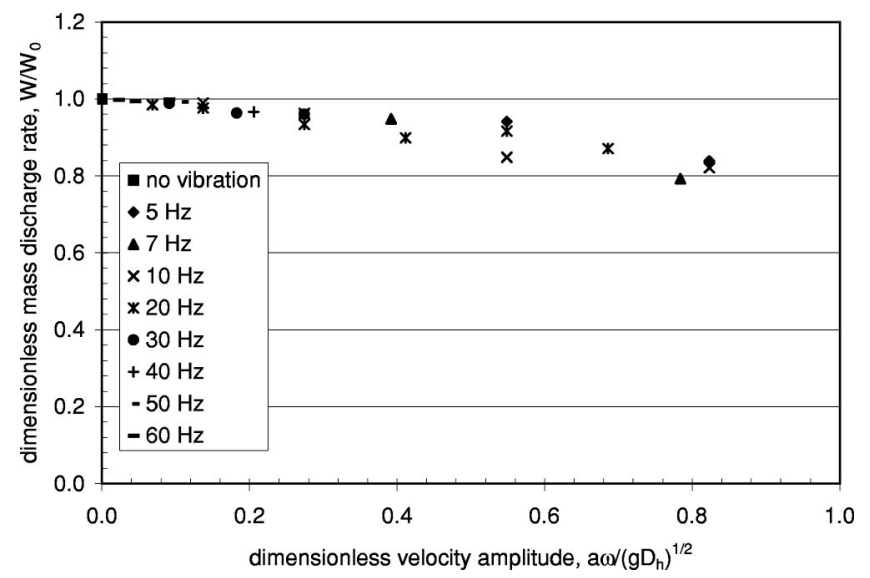

FIG. 12. Mass discharge rate from an oscillating hopper divided by the mass discharge rate from a nonvibrating hopper, $W / W_{0}$, plotted as a function of the dimensionless oscillation velocity amplitude, $a \omega /\left(g D_{h}\right)^{1 / 2}$. The plot only includes simulation data. 


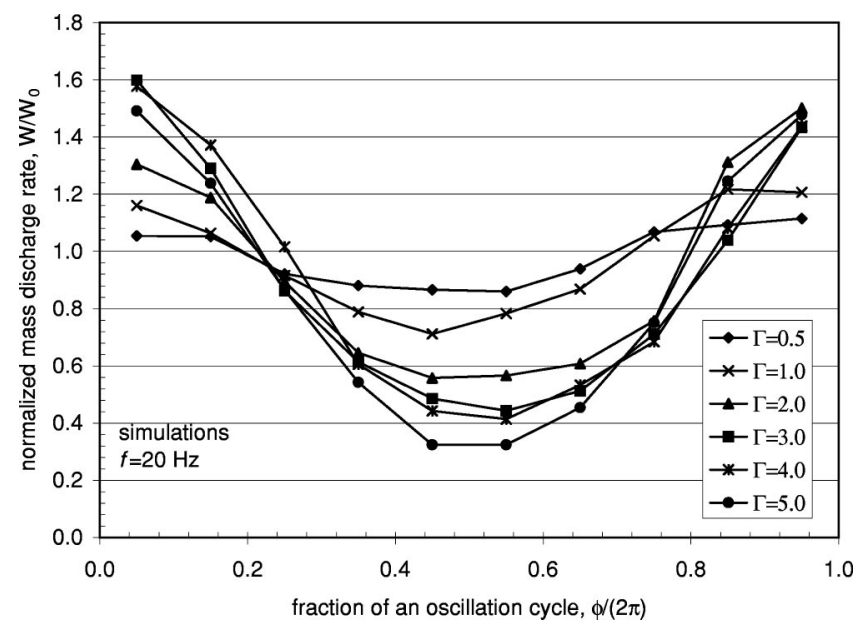

FIG. 13. The ratio of the mass discharge rate for a hopper oscillating at a frequency of $20 \mathrm{~Hz}$ to the mass discharge rate from a nonvibrating hopper, $W / W_{0}$, plotted against fraction of an oscillation cycle, $\phi /(2 \pi)$, for various dimensionless acceleration amplitudes.

hopper moves downwards $[0.25<\phi /(2 \pi)<0.75]$, the ratio is less than unity. For $\Gamma=0.5$ and 1.0 , the discharge ratio increases to values above unity as it moves upwards $[0.75$ $<\phi /(2 \pi)<1.0]$. For these two accelerations the discharge rate appears to roughly follow a cosine pattern with an amplitude that increases with vibrational velocity. For $\Gamma=2.0$ and 3.0, the discharge ratio deviates from a cosine wave, and the ratio remains below unity for a greater portion of the cycle.

In Fig. 14, similar results are shown for the normalized discharge rate at $60 \mathrm{~Hz}$ at $\Gamma=0.5,1.0$, and 2.0. The ratios appear to be slightly larger than unity as the hopper moves upwards, and at or slightly below unity for the remainder of the cycle. The cycle-averaged discharge rates are all within $10 \%$ of the discharge rate without vibration.

In addition to the variation of the mass discharge rate within the vibration cycle, it is also possible to determine the velocity of the particles exiting the hopper. Figure 15 pre-

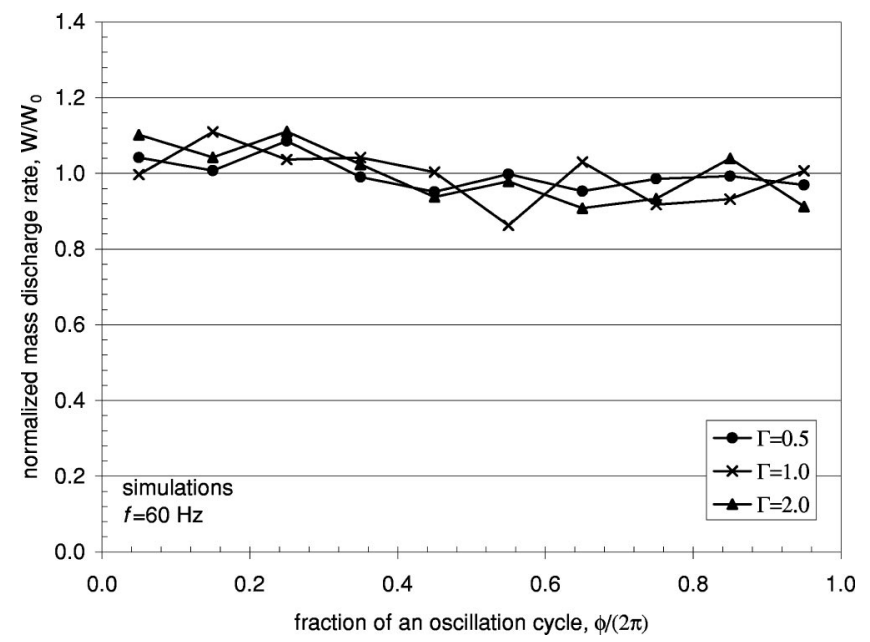

FIG. 14. The ratio of the mass discharge rate for a hopper oscillating at a frequency of $60 \mathrm{~Hz}$ to the mass discharge rate from a nonvibrating hopper, $W / W_{0}$, plotted against fraction of an oscillation cycle, $\phi /(2 \pi)$, for various dimensionless acceleration amplitudes.

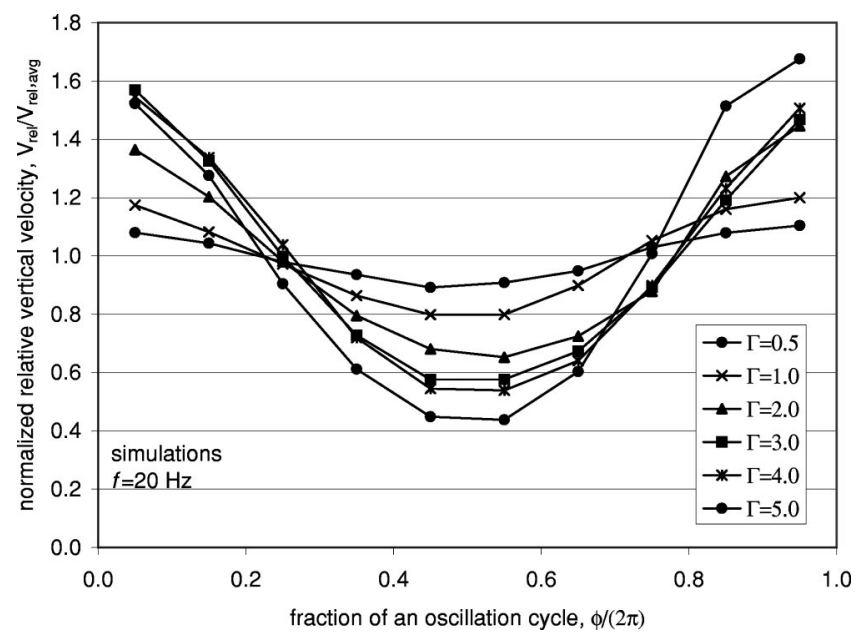

FIG. 15. The discharge velocity of particles relative to the hopper exit normalized by the cycle-averaged velocity, $V_{\text {rel }} / V_{\text {rel,avg }}$, as a function of phase angle, $\phi$, for a hopper oscillating at a frequency of $20 \mathrm{~Hz}$.

sents the vertical exit velocity relative to the hopper exit normalized by the cycle-averaged velocity as a function of phase angle for a frequency of $20 \mathrm{~Hz}$. The discharge velocity and mass flow rate show the same phase dependence. Hence, particles are leaving the hopper faster as the hopper is moving upwards and slower as the hopper is moving downwards. Figure 16, however, shows that the bulk density of the exiting material varies little over the oscillation cycle.

The average relative discharge velocity and bulk density for an oscillating hopper normalized by the average discharge velocity and bulk density for a stationary hopper are plotted in Figs. 17 and 18, respectively, as a function of the oscillation velocity amplitude. These figures indicate that the variations in the average mass flow rate due to vibration are influenced more by the variations in the bulk density than by the variations in the discharge velocity. For the parameters investigated the ratio of the bulk density for a vibrating hopper to the nonvibrating bulk density remains less than 1 with the smallest deviation occurring at small velocity amplitudes.

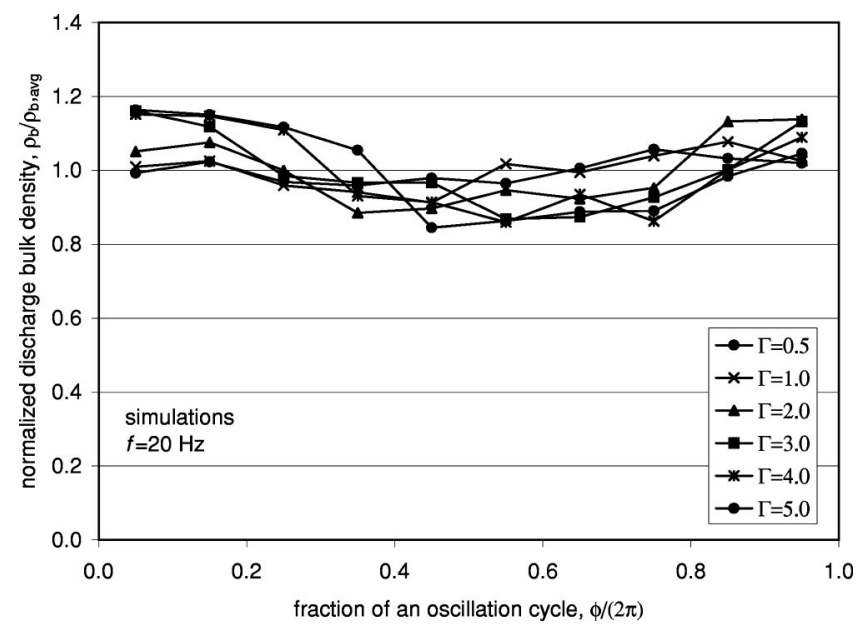

FIG. 16. The bulk density of particles at the hopper exit normalized by the cycle-averaged bulk density, $\rho_{b} / \rho_{\mathrm{b}, \text { avg }}$, as a function of phase angle, $\phi$, for a hopper oscillating at a frequency of $20 \mathrm{~Hz}$. 


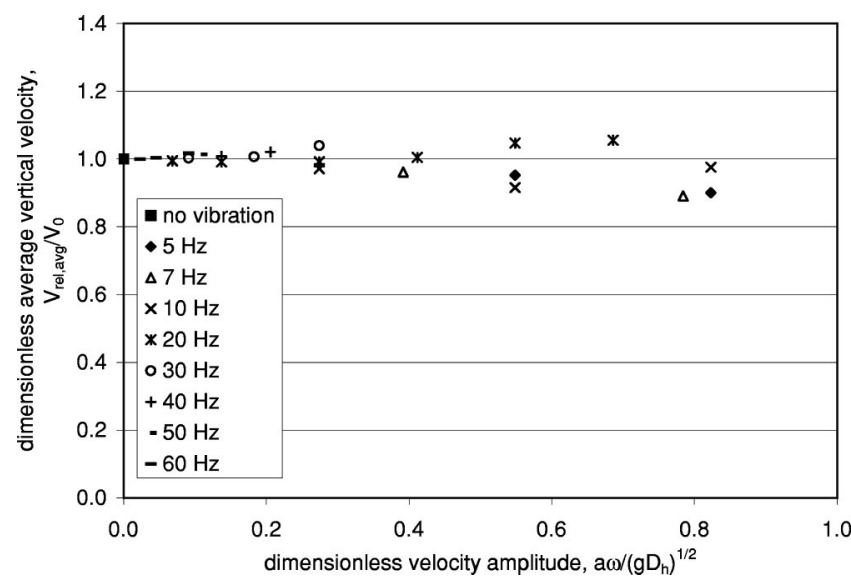

FIG. 17. Average discharge velocity (relative to the hopper exit) from an oscillating hopper normalized by the average discharge velocity from a stationary hopper, $V_{\text {rel,avg }} / V_{0}$, plotted as a function of the dimensionless oscillation velocity amplitude, $a \omega /\left(g D_{h}\right)^{1 / 2}$. The plot only includes simulation data.

The discharge velocity ratio, however, is less than 1 for frequencies less than approximately $20 \mathrm{~Hz}$ but is greater than 1 for frequencies greater than $20 \mathrm{~Hz}$.

Measurements of the horizontal position of particles and their velocities as they discharge from the hopper are also made with the simulations. The vertical velocity of particles relative to the hopper exit are shown in Fig. 19 for a hopper oscillating at $f=20 \mathrm{~Hz}$ and $\Gamma=2.0$. The data points are mass-averaged over 70 oscillation cycles. The discharge profile for a nonvibrating hopper is also included in the plot and is indicated by the thicker line. The vertical velocity relative to the hopper at discharge fluctuates in a manner consistent with the measurements in Fig. 15. Furthermore, it is observed that while the granular bed is in flight, the discharge profiles are more uniform than when the bed is in contact with the hopper walls. This occurs because the walls shear the particle bed at the discharge plane.

\section{DISCUSSION}

The simulation and experimental data show similar trends although the absolute values of the data are not the same. This discrepancy may be due to the fact that the simu-

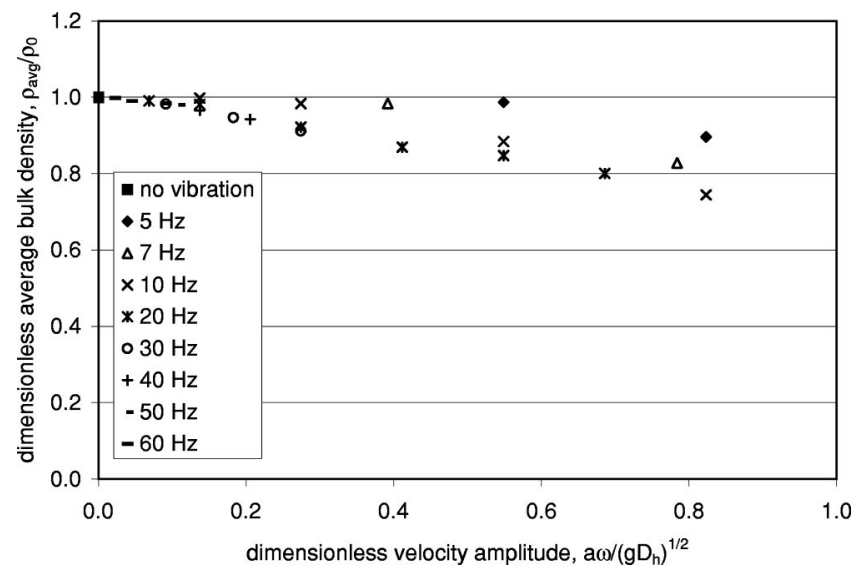

FIG. 18. Average discharge bulk density from an oscillating hopper normalized by the average bulk density from a stationary hopper, $\rho_{\text {avg }} / \rho_{0}$, plotted as a function of the dimensionless oscillation velocity amplitude, $a \omega /\left(g D_{h}\right)^{1 / 2}$. The plot only includes simulation data.

lations were two dimensional while the experiments were three dimensional. For a particular set of vibration parameters, $\Gamma$ and $f$, the instantaneous mass flow rate from the hopper closely follows the instantaneous velocity of exiting particles relative to the hopper (Figs. 13 and 15) while the bulk density of particles leaving the hopper varies little over an oscillation cycle (Fig. 16). However, for differing vibration conditions the average relative velocity remains nearly constant, less than $10 \%$ variation for the parameters investigated (Fig. 17), and instead it is the variation in the average bulk density that most affects the mass flow rate (Fig. 18). Note that although the strength of the convection cells varies with velocity amplitude, ${ }^{9}$ there is little influence on the discharge velocity from the hopper. Hence, the convection cells seem to play only a minor role on the mass discharge rate.

The data gathered in this study is also compared with the model proposed by Suzuki et al. ${ }^{4}$ in which the discharge rate is assumed to be a function of the instantaneous "effective gravity" acting on the particle bed. In their model, Suzuki et al. ${ }^{4}$ suggest that the instantaneous discharge rate from the hopper is given by

$$
W \propto \rho_{b} g_{\text {eff }}^{1 / 2} D_{h}^{5 / 2},
$$

where the effective gravity, $g_{\text {eff }}$ is given by

$$
g_{\text {eff }}=\left\{\begin{array}{l}
g[1-\Gamma \sin (\omega t)] \text { when the bed rests on the hopper walls, } \\
0 \text { when the bed is in flight, } \\
d \dot{y}_{\text {bed }} / d t \text { when the bed just impacts the hopper walls, }
\end{array}\right.
$$

where the acceleration acting on the bed at impact, $d \dot{y}_{\text {bed }} / d t$, is approximately equal to the difference between the floor velocity at impact and the particle bed free fall velocity just prior to impact divided by the duration of the impact period. Suzuki et $a l^{4}$ also propose that the only vibration parameter on which the bulk density of the material, $\rho_{b}$, should depend is the dimensionless acceleration, $\Gamma$, with the bulk density increasing when vibration is applied.

The data found in the experiments and simulations do not agree with the previously described model. The bulk density measurements presented here indicate that the bulk density has a dependence on both the frequency and acceleration amplitude and, furthermore, the bulk density decreases when vibration is applied. Suzuki et al. ${ }^{4}$ claim that the bulk density 


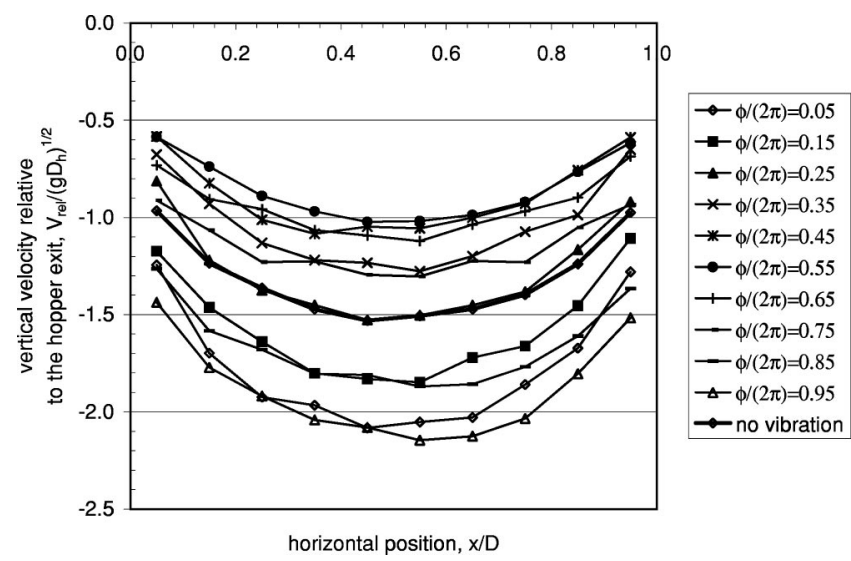

FIG. 19. The discharge velocity profile relative to the hopper exit at various phase angles for a hopper oscillating at $\Gamma=2$ and $f=20 \mathrm{~Hz}$.

of the material increases when the hopper is subject to oscillations. In addition to the differences with the bulk density observations, the relative velocity of the material leaving the hopper is different than what is proposed in the Suzuki et al. ${ }^{4}$ model. In their model the velocity of the exiting material is proportional to the square root of the effective gravity, $V_{\text {rel }}$ $\propto \sqrt{g_{\text {eff }}}$, where the effective gravity is given in Eq. (3). As shown in Fig. 15 where the velocity normalized by the cycleaveraged velocity is plotted as a function of oscillation phase angle for $f=20 \mathrm{~Hz}$, the effective gravity model proposed by Suzuki et $\mathrm{al}^{4}{ }^{4}$ is inaccurate in predicting the velocity of particles exiting the hopper. In particular, the assumptions that there is no discharge during the period when the bed is not in contact with the hopper walls and that there is a sudden surge of material when the bed impacts the hopper walls are clearly in error. In fact, the measurements reported here indicate that the effective gravity acting on the bed remains essentially constant and that the variation in the mass flow rate from the hopper is due to the movement of the hopper exit. As is shown in Fig. 17, the average discharge velocity varies little over the range of oscillation conditions investigated here. The variations in the average mass flow rate are thus due to variations in the average bulk density (Fig. 18).

The mechanism causing the slight increase in the experimental discharge rate at low velocity amplitudes remains unclear. One possibility is that the relative mobility of the material increases in this region while the density remains relatively unaffected. Indeed, the experimental measurements by Zik et al. ${ }^{15}$ show that the mobility of a particle in a vibrating bed is much greater when $\Gamma>1$ than for $\Gamma<1$.

It is interesting to compare the flow behavior in horizontally ${ }^{1}$ and vertically oscillated hoppers. Both types of vibration affect the hopper flow but in very different ways. For horizontal oscillations, small side-wall convection cells appear at the free surface of the particle bed near the hopper walls. Particles fall down along the hopper walls in the gap that opens up when the hopper moves away from the particle bed. The convection cells do not extend along the length of the wall as they do in vertically vibrated hoppers. A more striking difference between horizontally and vertically oscillated hoppers occurs in the discharge patterns and flow rates.
While the discharge pattern in a vertically vibrated hopper discharges more readily from the central core of the hopper, a horizontally oscillated hopper has an "inverted" pattern where material discharges more readily at the hopper walls. In addition, the mass discharge rate in a horizontally oscillated hopper increases with applied vibration in contrast to the decrease in mass flow rate observed with vertically oscillated hoppers. For both cases the discharge rate scales with oscillation velocity amplitude.

\section{CONCLUSIONS}

Granular flow from a vertically vibrating hopper was investigated. Particles within a closed hopper exhibit convection cells and surface waves similar to those observed in studies of horizontal beds. The convection cells are oriented such that particles move up along the inclined hopper walls and down at the hopper's center line. Observations from discrete element simulations indicate that the convective motion is a result of the dilation of the particle bed during free fall and interaction with the hopper walls.

The ratio of the discharge rate from a vibrating hopper to the discharge rate from a nonvibrating hopper decreases with vibration except at the highest frequencies. The discharge rate scales with the vibration velocity amplitude with a secondary dependence on the acceleration amplitude. The variation in the mass discharge rate during an oscillation cycle occurs primarily due to the relative velocity between the discharging material and the oscillating container. The bulk density of the material changes little during the oscillation cycle. The variations of the mass discharge rate for different oscillation parameters, however, is due to variations in the discharging material's bulk density. These findings do not support the model proposed by Suzuki et al. ${ }^{4}$ that accounts for the mass discharge rate trends using a variable effective gravity acting on the material within the hopper.

Although subjecting an entire hopper to vertical vibrations may not be practical in many applications, these results serve to demonstrate the significant effects that vibration can have on flow from a hopper. The paper also demonstrates the effect of vibration on the internal flow field. Although not studied in this paper, vertical vibration may prevent arching of a cohesive material due to the internal circulation of the material. $^{5}$

${ }^{1}$ M. L. Hunt, R. C. Weathers, A. T. Lee, C. E. Brennen, and C. R. Wassgren, "Effects of horizontal vibration on hopper flows of granular materials," Phys. Fluids 11, 68 (1999).

${ }^{2}$ C. R. Wassgren, C. E. Brennen, and M. L. Hunt, "Vertical vibration of a deep bed of granular material in a container," J. Appl. Mech. 63, 712 (1996).

${ }^{3}$ H. Takahashi, A. Suzuki, and T. Tanaka, "Behavior of a particle bed in the field of vibration. I. Analysis of particle motion in a vibrating vessel," Powder Technol. 2, 65 (1968).

${ }^{4}$ A. Suzuki, H. Takahashi, and T. Tanaka, "Behavior of a particle bed in the field of vibration. II. Flow of particles through slits in the bottom of a vibrating vessel," Powder Technol. 2, 72 (1968).

${ }^{5} \mathrm{~K}$. Lindemann and P. Dimon, "Two-dimensional granular flow in a vibrated small-angle funnel," Phys. Rev. E 62, 5420 (2000).

${ }^{6}$ J. B. Knight, H. M. Jaeger, and S. R. Nagel, "Vibration induced size 
separation in granular media: the convection connection," Phys. Rev. Lett. 70, 3728 (1993).

P. Evesque and W. Meftah, "Mean flow of a vertically vibrated hourglass," Int. J. Mod. Phys. A 7, 1799 (1993).

${ }^{8}$ R. M. Nedderman, U. Tüzün, S. B. Savage, and G. T. Houlsby, "The flow of granular materials-I. Discharge rates from hoppers," Chem. Eng. Sci. 37, 1597 (1982).

${ }^{9}$ C. R. Wassgren, "Vibration of granular materials," Doctoral thesis, California Institute of Technology, Pasadena, CA, 1997.

${ }^{10}$ F. Melo, P. Umbanhower, and H. Swinney, "Hexagons, kinks, and disorder in oscillated granular layers," Phys. Rev. Lett. 75, 3838 (1995).
${ }^{11} \mathrm{~J}$. B. Knight, "External boundaries and internal shear bands in granular convection,” Phys. Rev. E 55, 6016 (1997).

${ }^{12}$ E. L. Grossman, "Effects of container geometry on granular convection," Phys. Rev. E 56, 3290 (1997).

${ }^{13}$ A. Karion, "Couette flows of granular materials: Mixing, rheology, and energy dissipation," Doctoral thesis, California Institute of Technology, Pasadena, CA, 2000.

${ }^{14}$ P. A. Cundall and O. D. L. Strack, "A discrete numerical model for granular assemblies," Geotechnique 29, 47 (1979).

${ }^{15}$ O. Zik, J. Stavans, and Y. Rabin, "Mobility of a sphere in vibrated granular media," Europhys. Lett. 17, 315 (1992). 